\title{
Study Protocol Version
}

National Cancer Institute

\section{Source}

National Cancer Institute. Study Protocol Version. NCI Thesaurus. Code C93490.

A plan at a particular point in time for a formal investigation to assess the utility, impact, pharmacological, physiological, and/or psychological effects of a particular treatment, procedure, drug, device, biologic, food product, cosmetic, care plan, or subject characteristic. 